\title{
JD18: Quasar Cores and Jets - poster-paper summary
}

\author{
$\mathrm{J}$ V Wall \\ Department of Physics \& Astronomy, University of British Columbia, \\ Vancouver, BC V6T 1Z1, Canada
}

\section{Introduction}

Of the 17 poster papers presented at IAU25 Joint Discussion 18, three (1821, $1824,1834)$ are essentially theoretical, three $(1828,1829,1831)$ deal with polarization, screens and absorbers, one (1818B) considers galaxy formation and evolution, $7(1817,1820,1826,1830,1835,1836,1837)$ describe source structure and spectra, and three $(1812,1818,1823)$ deal with Intra-Day Variability (IDV).

\section{Theory}

Paper 1821, Microphysics of AGN central engines by Zdenka Kuncic explores the energetics and dynamics of accretion-disc coronae in AGN. A solar analogy is used to identify the mechanisms: bulk plasma heating, particle acceleration, bulk kinetic energy. Spectral signatures from the radiative cooling processes are presented. A particularly useful feature of this paper that it lays out all the physical processes involved.

Y. Lu, K.S. Cheng and S.N. Zhang in poster 1824 describe $A n$ accretion model for the growth of the central black hole. Their model, requiring but two parameters, shows how ionization instability produces an S-curve in the $\log$ (mass) vs $\log$ (surface density) plane. The bottom branch of the S-curve is ADIOS, advection dominated accretion, while the upper branch is rapid accretion and represents the QSOs we see now. The bulge-mass relation reported by Magorrian et al (1998) arises naturally in the model. However, time scales are such that low-mass black holes may have to grow with a different (faster) process if the accretion rate is Eddington-limited.

Paper 1834 by S. Ramotholo considers Possible optical identification of gamma-ray blazars, pointing out that detection is important in understanding particle acceleration processes. With missions HESS, GLAST, and MAGIC coming it is important to consider efficient detection methods. The peak position of synchrotron emission is crucial in determining whether a blazar will be seen at $\mathrm{GeV}$ or $\mathrm{TeV}$ energies. The paper is a combined theoretical and observational study suggesting how to determine this peak from optical observations.

\section{Polarization, screens and absorbers}

In paper 1828, Nakai Naomasa considers Water maser disks in $A G N$ - two types of cold absorber. The objects in question are $\mathrm{H}_{2} \mathrm{O}$ megamasers and the two types are distinguished by the flux ratio of high-velocity to systematic $\mathrm{H}_{2} \mathrm{O}$ 
features. The former (prototype NGC 4258) shows weak or no Fe K $\alpha$ lines; the latter (prototype NGC 1068) strong $\mathrm{Fe} \mathrm{K} \alpha$ lines. The model has the effect caused by the former having thin disks, the latter thick disks - and VLBI observations have now revealed these disks. The paper has an excellent illustration of how the Fe fluorescence works with the two disk types to give the dichotomy in line-strength ratios.

VLBA polarimetry: a Faraday screen in 3C273, paper 1829 by R. Ojha et al., describes tracking the polarization of $3 \mathrm{C} 273$ 's superluminal radio components with VLBA over 6 epochs at 2-month intervals. The fractional polarization increases as a component moves out. It could be that the field becomes more ordered with distance; or that the components are moving out from behind a screen blocking polarized radiation. The observations favor the latter - but the drawback is that this now requires some kind of filamentary Faraday screen producing the same optical depth at 15 and $22 \mathrm{GHz}$, with little Faraday rotation.

W. Cotton (paper 1831) describes Evidence for a low filling factor in the NLR of CSS QSOs. The Compact Steep-Spectrum (CSS) sample of Fanti et al (2001) has complete linear-size measurements from VLBA observations. A plot of integrated polarization (from the NVSS) against size shows an absence of polarization at sizes $<6 \mathrm{kpc}$. Detailed examination of $3 \mathrm{C} 43,3 \mathrm{C} 138$, key sources in the sample, shows that some weakly polarized emission remains, despite the known amounts of ionized gas in Narrow Line Regions (NLR). The implication is that the NLR plasma has a low filling factor but high covering factor. A model in which the plasma is arranged in sheets will work, and is testable by looking for Faraday rotation at high frequencies.

\section{Galaxy evolution}

A possible Radio - X-ray connection in high-z quasars on the sub-kpc scale is discussed in 1818B by A.P. Lobanov, L.I. Gurvits and S. Frey. VLBA observations have revealed unusually prominent sub-kpc-scale jets at rest-frame frequencies of $7.2 \mathrm{GHz}$ (with VLBA) and $25 \mathrm{GHz}$ (with VSOP) for the QSOs $2215+020$ and $1713+218$ respectively. Both have the jets pointing toward ROSAT X-ray extensions. Both have redshifts of $\sim 4$. There are 71 QSOs in the current sample with $z>3$; the authors suggest that such objects may serve as beacons for protoclusters in the early universe, providing a resource for tracing galaxy and structure development.

\section{Spectra and Structure}

N. Gizani and M. Garret consider Jet misalignment in radio galaxies - the unification theory (paper 1817). The radio sources Her A and 3C 310 are FRI/II objects of large overall double structures (arcmin scales) with ring-like features around the nucleus. The structures on VLBI scales show relatively high misalignments with the axes of the huge outer lobes of these objects. At face value this lack of alignment is troubling for unified models, which prescribe that central jet structures should lie along the ejection/lobe axis. The authors point out that the objects resemble CSS-class QSOs in this misalignment, and a relatively high-density ISM (interstellar medium) is ascribed to CSS QSOs to explain their 
small size by confinement of the radio lobes. If by analogy Her A and 3C 310 also have high density ISMs, then their misalignments present much less of a challenge to unified models.

$X$-ray measurement of energetics in lobes of radio galaxies is discussed in paper 1820 by Naoki Isobe. Measurements with ASCA, Chandra and XMM for a small sample of radio galaxies have mapped diffuse X-ray emission from the lobes of these radio galaxies, due to Inverse Compton scattering of CMB photons by the synchrotron electrons of the lobes. The X-ray and synchrotron radiation was compared in order to measure energy densities $U_{e}$ and $U_{m}$. The following results were obtained: (1) $U_{e} \sim 10 U_{m}$; (2) $U_{e}$ in lobes $\propto$ luminosity of nucleus, shown to follow if jet-luminosity $\propto$ accretion; (3) $U_{m}$ does not depend on luminosity of the nucleus; and (4) in several lobes, $U_{e}$ is uniform but $U_{m}$ is higher towards the lobe periphery.

The expanding lobe of $3 C 84$ is the subject of paper 1826 by K. Asada et al. (Note that $3 \mathrm{C} 84$ is Per A, NGC 1275, etc; $\mathrm{z}=0.0172$, with extended structure dominated by the bright southern lobe.) Asada et al. have made multiepoch observations with VSOP/HALCA, the VLBA and the full VLA; the paper presents superb maps. There is a monotonic decrease of flux, presumably due to adiabatic expansion; the southern lobe is going south at $0.3 \mathrm{c}$; and some of the knots within the lobe are in motion while others are stationary. It appears as though the jet is hitting/pushing the inner edge (the northern top) of the main lobe.

A major observational program is sketched out in paper 1830 by $\mathbf{R}$. Ojha et al., which presents Results from 8.4 GHz VLBI imaging of 184 southern QSOs. This is the single largest sample of QSOs studied with VLBI; many of these QSOs have never before been examined at milliarcsec resolutions. The VLBI network used in the survey comprises the Australia Long Baseline Array together with telescopes in South Africa and Hawaii. Some early maps are shown - only half the data have been reduced, but from this it appears that $\sim 50 \%$ show extended structures on milliarcsec scales and $\sim 10 \%$ are doubles. There is much to come from this extensive observing program.

Zhi-Qiang Shen, J.M. Moran and K.I. Kellermann describe $A$ bent superluminal jet in the central parsecs of OV-236 (paper 1835). OV-236 is PKS 1921-293, with $\mathrm{z}=0.325$ at which 1 millarcsec $=4.6 \mathrm{pc}$; the object is compact, variable and bright at $\mathrm{mm}$ wavelengths. Multi-epoch multi-frequency VLBA observations were carried out at 5 and $43 \mathrm{GHz}, 1994$ to 2002; additional VLBA observations were made at 12,15 and $86 \mathrm{GHz}$. In two VLBA images of OV-236 obtained simultaneously at 5 and $43 \mathrm{GHz}$ in 2000 , a misalignment of about $70^{\circ}$ can be seen in the overall structure. The central 1 pc region consists of two equally compact $(\sim 0.1$ milliarcsec) components whose relative position appears unchanged in all 7 -epoch $43 \mathrm{GHz}$ images over the 8 years. It is possible that they are associated with a massive binary black hole. A schematic model is drawn to show how the jet emission observed at different scales may be explained with a single smoothly-curving trajectory.

The Radio spectra of the Parkes half-Jansky FS sample are considered by O.I. Wong and R.L. Webster in paper 1836. ATCA flux-density measurements were made simultaneously at $1.4,2.5,4.8$ and $8.4 \mathrm{GHz}$ of the 323 sources in 3.9 sterad above $S_{2.7 \mathrm{GHz}}=0.5 \mathrm{Jy}$. From these, the radio spectral-energy dis- 
tributions (SEDs) were characterized. Comparison of results at different epochs showed that most SEDs move up and down flux scales at each frequency without changing spectral type. The observations permit consideration of a number of issues, e.g. long-term variability studies, development of simple models of synchrotron mechanisms, and comparison with optical and IR SEDs.

The same sample is the subject of paper 1837: Jet vs disc: the optical output of FSRQ by M. Whiting et al. The authors carried out BVRIJHK photometry for the Parkes 0.5-Jy sample. They draw a number of conclusions from decomposition of the SEDs, considering the accretion disk to emit as a (blue) power-law and the relativistic jet as a (redder) synchrotron bump. Evidence for synchrotron emission is shown by $40 \%$ of the objects, while $40 \%$ are best fit by a single power law. BL Lac objects are best fit with disc+jet model; the relatively weak emission lines of BL Lacs are thus not wholly due to swamping by a boosted jet component. The disc and broad-line region emission appears intrinsically weak, perhaps due either to a different accretion process, or to a greater amount of outflow into the jet disrupting inner regions of the disk.

\section{Intra-Day Variability}

Two poster papers with authorship in common consider J1819+3845, the most variable QSO: 1812, Structure of the IDV QSO J1819+3895 from 1 microarcsec to 1 milliarcsec by G de Bruyn, J-P Macquart and J Dennett-Thorpe, and $1818, J 1819+3845$ as seen by VSOP by L I Gurvits, G de Bruyn and J Dennett-Thorpe. The former describes combined VLBI and IDV observations and simultaneous multi-frequency modeling of the source structure, presenting the results in a fine schematic diagram. Structure scales from 100 nanoarcsec to 1 microarcsec for the scintillating component are implied, with the corresponding requirement that $T_{b}$ exceeds $10^{15} \mathrm{~K}$. The latter describes detection of J1819+3845 on all 78 baselines of a VLBI - HALCA experiment, with analysis using closure phases and a novel "VLBI-scintillation delay" technique.

Paper 1823 by J. Lovell et al. presents the First results from MASIV, the Micro-Arcsecond Scintillation-Induced Variability survey. In the authors' words, this is 'a $5-\mathrm{GHz}$ VLA survey of the northern sky to obtain $100-150$ scintillating flat-spectrum extragalactic sources with which to examine both the microarcsecond structure and parent populations of these sources, and to probe the turbulent interstellar medium responsible for the scintillation. Early findings: (1) The fraction of sources that scintillate and their fractional scintillation amplitude both increase for the weaker $(\sim 100 \mathrm{mJy})$ sources; $(2) \sim 20 \%$ of compact flat-spectrum sources vary on at least one epoch; (3) $\sim 50 \%$ show annual cycles; (4) Non-isotropic sky distribution indicates that the majority of these are tied to the ISM. A vast data-base will result from this major project, and these first results are the forerunners of much to come.

The poster papers represented a vital and integral part of Joint Discussion 18 and the organizers are very grateful to the authors for their efforts. 